\title{
A Measurable Model of the Creative Process in the Context of a Learning Process
}

\author{
Min $\mathrm{Ma}^{1,2}$, Fred Van Oystaeyen ${ }^{1}$ \\ ${ }^{1}$ Department of Mathematics and Computer Science, University of Antwerp, Belgium \\ ${ }^{2}$ Department of Psychology, Central University of Finance and Economics, China \\ Correspondence: Min Ma, Department of Psychology, Central University of Finance and Economics, China
}

Received: June 12, 2015 Accepted: June 26, 2015 Online Published: October 19, 2015

doi:10.11114/jets.v4i1.1152 URL: http://dx.doi.org/10.11114/jets.v4i1.1152

\begin{abstract}
The authors' aim was to arrive at a measurable model of the creative process by putting creativity in the context of a learning process. The authors aimed to provide a rather detailed description of how creative thinking fits in a general description of the learning process without trying to go into an analysis of a biological description of the brain activity. The tool was the aspect deformation process of an indicator process partially ordered by causality. The building blocks of the process formed a set of indicators. An indicator was represented as a set of aspects and enough aspects are discerned in order to have a good characterization of the indicator. As an application of the model and theories, we described some implications for didactics as well.
\end{abstract}

Keywords: aspect deformation process, creative process, fantasy object, indicator, shadow object

\section{Literature Review: Models of Creativity and Creative Process}

Creativity is a vast and multidisciplinary subject of investigation and perhaps there as are many points of view as there are researchers. Rhodes (1961) classified four aspects for creativity: Person, Process, Product, and press/environment. In trying to understand creativity many models are constructed. Some paid more attention to the analysis of creative thinking (Davidson \& Sternberg, 1984; Sternberg \& Davidson, 1982), while others explored the phases in creative thought, such as the Geneplore model (Finke, Ward, \& Smith, 1992). However, these models merely limited to one or two aspects (creative insights, creative thought) of creativity. Admittedly, there are some theories or models concluded or constructed from a point of global view, for instance, Sternberg (2006)'s investment theory, which took into consideration six aspects: intellectual skill, knowledge, thinking style, personality, motivation and environment; and a propulsion theory of creative contributions (Sternberg, 2006), which divided creative contributions into three categories: types of creativity that accept current paradigms and attempt to extend them, reject current paradigms and attempt to replace them, and a type of creativity for synthesis of current paradigms. The ingredients in these theories are properties or effects of the creative actions not necessarily fitting in a theory dealing with the evolving of creativity in the thinking process. Alongside of the research of factors of creativity as stated above, stages of creative process were also explored. Graham Wallas proposed a four-stage model to describe creative process: preparation, incubation, inspiration, and verification (Rhodes, 1961). Mumford and Gustafson (1988) gave an outline of creative process research from three aspects: production process, association process and unconscious process. Ruscio, Whitney, and Amabile (1998) proposed a rather elaborated model of the creative process, which takes into account three creativity components, intrinsic motivation, domain-relevant skills and creativity-relevant processes. Again these models rather describe a posterior observation of the superficial structure of the process in terms of activities that do not always happen in a structural way in the process. There is a need for a global theory putting the creative process in the framework of a learning process, described by fitting ingredients plus their interrelations, with specific cognitive activities making it into a creativity process. This should be done with unambiguous definitions and a transparent logical structure of the process but allowing for the personal traits involved in the creative thinking. The model we aim to construct should fit also existing research, for example, we will integrate some factors of person, product and press in a novel way. The creative process is of course personal but its structure will be generic, it is a learning process turning into an investigation process leading to creativity. The definition of creativity in our research is based on the trait of originality and also hinges on the social recognition in a social context. Thus, our research questions are: 
What is a step-by-step creative process? How to construct a model including a large amount the ingredients related to creativity and further make these ingredients well structured How to apply this model in didactics?

Our model of the creative process can be well understood from three perspectives. Firstly, we proposed the process model that was constructed based on the tool of the aspect deformation process of an indicator process partially ordered by causality. Secondly, we described the creative process using specific indicators represented by a cluster of aspects and relations among indicators induced from an aspect relation. These indicators and aspects can be verified and revised by some methods (e.g., confirmative factor analysis). Thirdly, as the most pragmatic part, our creative process model has some implications for didactics. This well-constructed paradigm leads to better understanding between students and teachers, allowing to improve the quality and efficiency of a learning process.

We put creative process in the context of a learning process. Without discussion the learning process we cannot clearly articulate the nature of the creative process. Kaufman and Beghetto (2009) proposed the four C model of creativity. They underscored that besides everyday creativity ("little-c") and eminent creativity ("Big-C") (Beghetto \& Kaufman, 2007), creativity inherent in the learning process ("mini-c") and progression beyond little-c that represents professional-level expertise ("Pro-c") should be considered. They also pointed out that "mini-c" was "designed to encompass the creativity inherent in the learning process" and defined as "the novel and personally meaningful interpretation of experiences, actions, and events" (Beghetto \& Kaufman, 2007, p. 3). These notions pertaining to learning process and personal interpretation fit our ideas well. Our research aimed to expand on the creative process in the context of learning process. However, a slight dissent is that we assume that the process inducing "mini-c" is a general process running through the processes of generations of creativities as they classified. In other words, In fact we believe that the basic creative process is the same for each of the four $\mathrm{C}$, the difference being either in associating levels (everyday, eminent) or context factors (learning, professional). So we put main emphasis on creative interpretation, general creativity process, without differentiating via levels or context factors. The creative process is an effective learning process if we understand the creative process as a personal interpretation and construction process.

\section{A Measurable Model for the Creative Process}

\subsection{Interpretation of Observation: The Shadow Object}

An observer begins the study of the object by observing it. The nature of the object determines which senses can be effectively used during the observation. Perhaps it is an object in space and it is first visually perceived, perhaps it is a series of symbols, letters, words... or a text that provides us with a description of an object we are unable to observe with our senses. An object of the latter type will be called an "abstract object". In fact our theory concerning the aspect deformation in a learning process is based on experience in mathematical research. So in general, the observed object is not completely known nor a faithful representation of the real object, in fact one may argue philosophically that the same object cannot be observed more than once, because everything changes in time and observation takes time. At the end of the learning process on an object of study (abstract or real) a new abstract object has been formed in the brain of the observer, let us call it the shadow object. In the study process at each stage there will be a temporary shadow object reflecting certain properties of the real object; this temporal object is continuously adjusted further by well-aimed questions and thought experiments or actions taken in reality like experiments, leading to new observations building finally the shadow object. The object studied is called an ingredient of the learning process and in the completion of the process in time the ingredient is replaced by a collection of new observations and ideas we call the aspects of the ingredient; at the end of the process the ingredient is viewed as being adequately described by the aspects. Different aspects of an ingredient relate to different ways of thinking, drawing from existing experience, or from the data in the memory, but also from abstract fantasies. The stream of temporary shadow objects, enriched with other ideas and images, even stemming from a priori unrelated objects, creates finally new study items of abstract nature that we may call "relations" between aspects. A process is a set of ingredients with a relation (usually a partial order, for example causality), where to each ingredient there is associated a finite set of aspects with a relation on the whole set of aspects; later we will formalise this definition and define an aspect relation more concretely. This model will provide us with a tool to analyze and define a learning or creative process allowing the introduction of numerical parameters that will then lead to a measurable model via evaluation processes.

\subsection{A Deformation of a Partially Ordered Process (See Figure 1)}

The creative process is based on the manipulation of abstract ideas, stemming from the observations of the reality, leading to fantasy objects that may in turn be realized in some ways. The extra depth in the manipulation of ideas and the creation of fantasy objects related to the information is thereby allowing more creative thinking. The thinking related to a creative activity is not linearly ordered in time to provide a measurable model for the creative process based on a deformation of a partially ordered (e.g. by causality) process in time. 


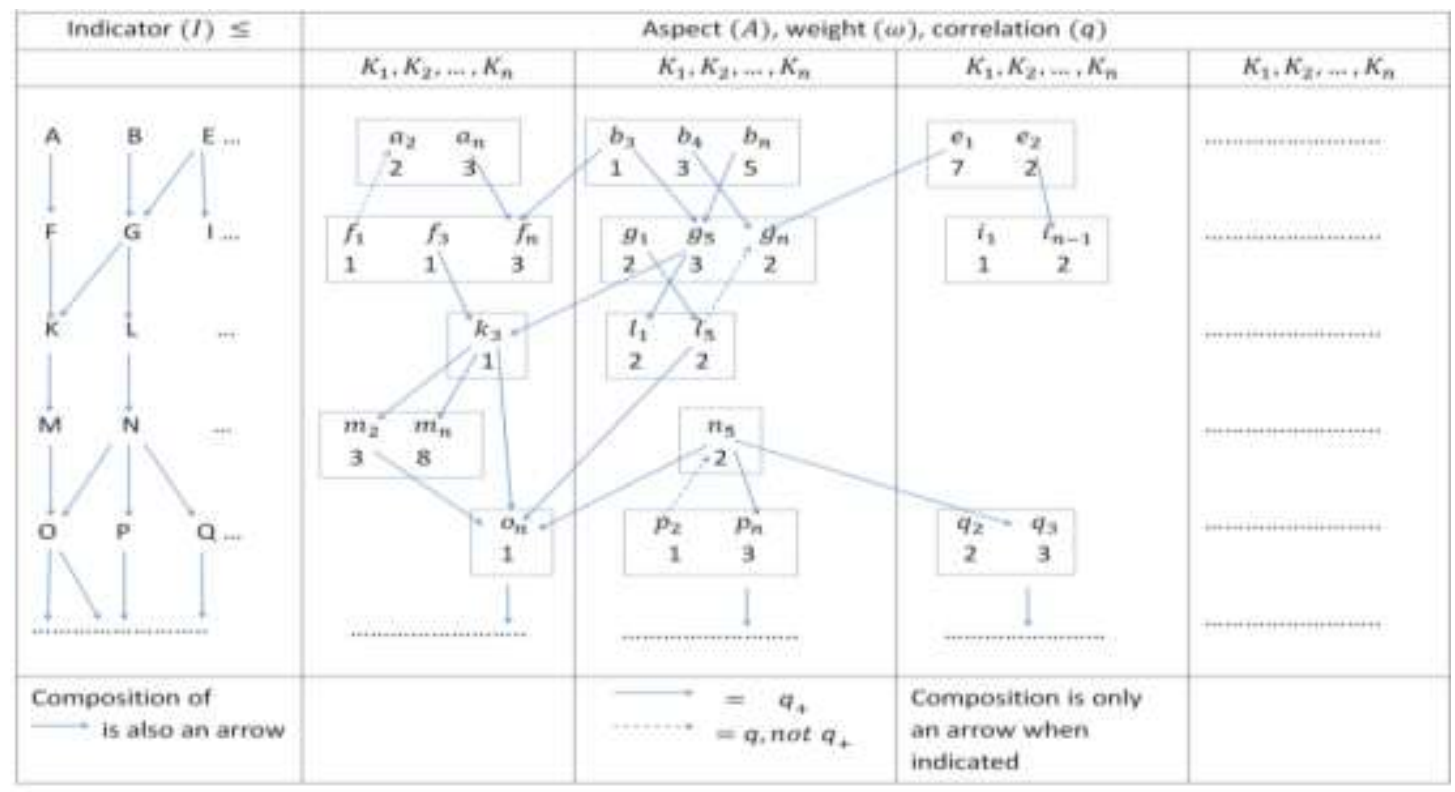

Figure 1. A measurable Model for the Process with Focus on Mathematics. In Figure 1, $\mathbf{q}_{+}$is the relation on A coming from deleting all arrows between elements of A not corresponding to an underlying arrow of $\mathbf{I}$. This called the "partial" relation. In the above picture the relation on $\mathbf{I}$ is a partial order relation, so we did not draw the composition of arrows as an arrow.

Both the learning process and the creative process are processes defined in time. We view them by looking back at them when a certain endpoint or observation point has been reached. So we describe the process (or part of it) in a finished state and then try to understand how the process evolves in time filling out the final picture. The objects necessary to describe a process are called (process-) indicators; let I be the collection of indicators describing a process P. We have to describe each indicator by its aspects; there should be enough well-defined aspects to obtain a narrow description of the indicator. The collection of all aspects of all ingredients of $\mathrm{P}$ is denoted by A. Every indicator $\mathrm{A}$ in $\mathrm{I}$ has a collection of aspects A (A) describing it. We assume that an aspect describing $\mathrm{A}$ is not identical to one describing a different $\mathrm{B}$ in $\mathrm{I}$; this can always be arranged by adding to an aspect describing $\mathrm{B}$ some information about the connection to $B$, so this assumption is harmless. We define a map $\pi: A \rightarrow$ I by putting $\pi(a)=A$ if a $\in A(A)$; since $A(A)$ and $A(B)$ for $A \neq B$ have nothing in common, $A(A) \cap A(B)$ is empty. This map is well-defined and surjective (that is for every $A$ in $I$ there is an a $\in A$ such that $\pi(a)=A$ ). The logical structure of the process is encoded by a relation $q$ defined on $A$. The product collection $A \times A$ is the collection of ordered pairs $(a, b)$, for $a$ and $\mathrm{b}$ in $\mathrm{A}$, a relation $\mathrm{q}$ is just a subset of $\mathrm{A} \times \mathrm{A}$. We say that $\mathrm{aqb}$ if $(\mathrm{a}, \mathrm{b})$ is in $\mathrm{q}$, we also write this $\mathrm{as} \mathrm{a} \rightarrow \mathrm{b}$. A typical example is the causality relation where $a \rightarrow b$ means that $b$ "follows" from $a$. The A (A) for A varying over I is a partition of A, i.e. every a in $\mathrm{A}$ is in exactly one $\mathrm{A}(\mathrm{A})$ for some $\mathrm{A} \in \mathrm{I}$.

Definition: a relation $\mathrm{q}$ on $\mathrm{A}$ is called an aspect relation if:

$\mathrm{q}$ is reflexive, i.e. for all a in $\mathrm{A}$, aqa.

$\mathrm{q}$ separates indicators, i.e. if aqb for $\mathrm{a}$ and $\mathrm{b}$ in $\mathrm{A}$ then either $\mathrm{a}=\mathrm{b}$ or $\mathrm{a} \neq \mathrm{b}$ and then $\mathrm{aqb}$ excludes that $\mathrm{a}$ and $\mathrm{b}$ belong to the same indicator.

To an aspect relation we would like to associate a relation induced on I. We have a system of arrows between the collections A (A) and A (B), some from elements of A (A) to A (B), some in the reverse direction. If there are more arrows from $A(A)$ to $A(B)$ for example, then we could decide that $A \leq B$, defining a relation $\leq$ on $I$. However, some aspects in the description of an indicator are possible more important than others, so perhaps a weighing of this importance should be integrated in the definition of induced relation. An external weight $\omega: \mathrm{A} \rightarrow \mathbb{R}_{+}$, associates with each a in A a positive real number $\omega(\mathrm{a})$; we can also define weights with "percentages" or probabilities. The bruto weight of an indicator $\mathrm{A}$ is then

$$
\sum_{a \in \mathrm{A}(A)} \omega(a)
$$

and we denote this by $\omega(A)$ (where $a \in A(A)$ means $a$ is in the collection A (A)).

The nature of the weights determines the "exactness" of the process analysis. For an aspect relation q we define the 
graph weight as follows. Look at a subset $\mathrm{S}$ in $\mathrm{A} \times \mathrm{A}$ and look at the intersection $\mathrm{S} \cap \mathrm{G}(\mathrm{q})$ (i.e. the elements in $\mathrm{S}$ that are also in $G(q))$ where $G(q)$ is the collection of $(a, b)$ in $A \times A$ such that aqb. Define

$$
\omega_{\mathrm{q}}(S)=\sum_{\substack{(a, b) \text { in } \mathrm{a} \\ \mathrm{a} \rightarrow \mathrm{b}}} \omega(\mathrm{a}) \omega(\mathrm{b}) / \sum_{(\mathrm{a}, \mathrm{b}) \text { in } \mathrm{S}} \omega(\mathrm{a}) \omega(\mathrm{b})
$$

In case $\mathrm{S}=\mathrm{A}(\mathrm{A}) \times \mathrm{A}(\mathrm{B})$, where $\mathrm{A} \neq \mathrm{B}$ in $\mathrm{I}$, then:

$$
\omega_{\mathrm{q}}(\mathrm{A}(\mathrm{A}) \times \mathrm{A}(\mathrm{B}))=\sum_{\mathrm{aq} b} \omega(\mathrm{a}) \omega(\mathrm{b}) / \omega(\mathrm{A}) \omega(\mathrm{B})
$$

Because, as A (A) and A (B) are disjoint:

$$
\sum_{a \in A, b \in B} \omega(a) \omega(b)=\left(\sum_{a \in A} \omega(a)\right)\left(\sum_{b \in B} \omega(b)\right)=\omega(A) \omega(B)
$$

For $S$ as above, let $S^{0}$ be the set of $(x, y)$, such that $(y, x)$ is in $S$. The diagonal weight $\delta_{\mathrm{q}}(S)$ is then defined as

$$
\delta_{\mathrm{q}}(\mathrm{S})=\omega_{\mathrm{q}}(\mathrm{S})-\omega_{\mathrm{q}}\left(\mathrm{S}^{\circ}\right)
$$

Now for $\mathrm{x}, \mathrm{y}$ in $\mathrm{I}$ we define a new relation $\leq$ on $\mathrm{I}$ by putting $\mathrm{X} \leq \mathrm{Y}$ if and only if $\delta_{\mathrm{q}}(\mathrm{A}(\mathrm{X}) \times \mathrm{A}(\mathrm{Y})) \geq 0$, i.e. exactly if

Or also:

$$
\omega_{\mathrm{q}}(\mathrm{A}(\mathrm{X}) \times \mathrm{A}(\mathrm{Y})) \geq \omega_{\mathrm{q}}(\mathrm{A}(\mathrm{Y}) \times \mathrm{A}(\mathrm{X}))
$$

$$
\sum_{\mathrm{x} \rightarrow \mathrm{y}} \omega(\mathrm{x}) \omega(\mathrm{y}) \geq \sum_{\mathrm{y}^{\prime} \rightarrow \mathrm{x}^{\prime}} \omega\left(\mathrm{y}^{\prime}\right) \omega\left(\mathrm{x}^{\prime}\right)
$$

Where $x, x^{\prime}$ in $X$ and $y, y^{\prime}$ in $Y$ and $x \rightarrow y$ means $x q y$. In case all weights are equal, e.g. $\omega(a)=1$ for all a in $A$, then $\left(^{*}\right)$ just means that there are more (or equal) arrows from elements in $X$ to elements in $Y$ than conversely.

The relation $\leq$ is reflexive, i.e. $\mathrm{A} \leq \mathrm{A}$ for $\mathrm{A}$ in $\mathrm{I}$. Usually we start from a relation $\leq$ on $\mathrm{I}$ that defines a partial order (like causality); this means that $\leq$ is anti-symmetric ( $x \leq \mathrm{y}$ and $\mathrm{y} \leq \mathrm{x}$ means $\mathrm{x}=\mathrm{y}$ ) and transitive ( $\mathrm{x} \leq \mathrm{y}$ and $\mathrm{y} \leq \mathrm{z}$ means $\mathrm{x} \leq \mathrm{z}$ ). It can be shown that a partial order on I, say $\leq$, can be lifted to an aspect relation that is reflexive and anti-symmetric but not necessarily transitive.

In case an aspect relation $\mathrm{q}$ is such that the induced relation on I is a partial order then we call the relation $\mathrm{q}$ indicator ordering, for our application we shall describe a process by a partially ordered set of indicators, ordered by causality and an aspect deformation of this by describing aspects and an indicator ordering aspect relation (hence reflexive and anti-symmetric but not necessarily transitive).

The choice of causality as the underlying ordering is a very natural one for all human activities, however, it is very meaningful that we allow a deformation relation in the description of the more detailed aspect processes because the development of human ideas in the brain is not completely dictated by causality. Another way of ordering of ideas does not reflect the logical structure of the observed material and this gives rise to a version of the "uncertainty principle" (like the Heisenberg principle in quantum mechanics also follows from the order of measurements). On the level of indicators the fact that the chronological cognitive digestion does not follow the logical causal structure is not a big problem because exactly the order given by the causality can be easily recognized making the exact structure clear. However, for "understanding" we need to know the micro-structure of the process. i.e., the aspect relation and the cluster-structure of the aspect description; this micro-structure is not necessarily causal (the aspect relation need not be a partial ordering) and so knowing it by chronological analysis requires a deeper analysis of the clusters around any giving aspect. This is the origin of several problems in didactics where one wants to convey understanding and not just factual knowledge. The teacher has to understand the complexity of the matter and has to know exactly where the student fails to "understand" the reasoning on the aspect level. 


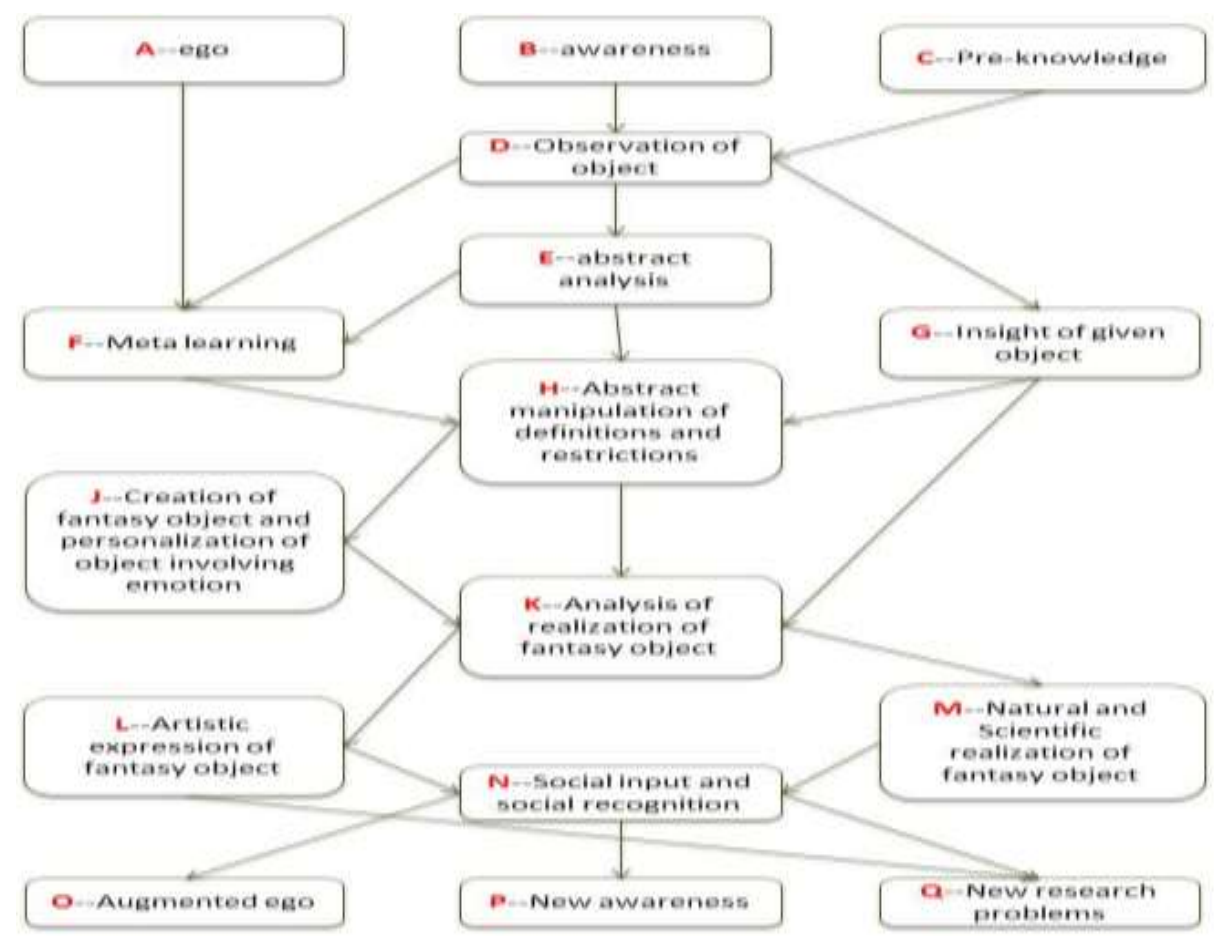

Figure 2. Indicators of the creative process model.

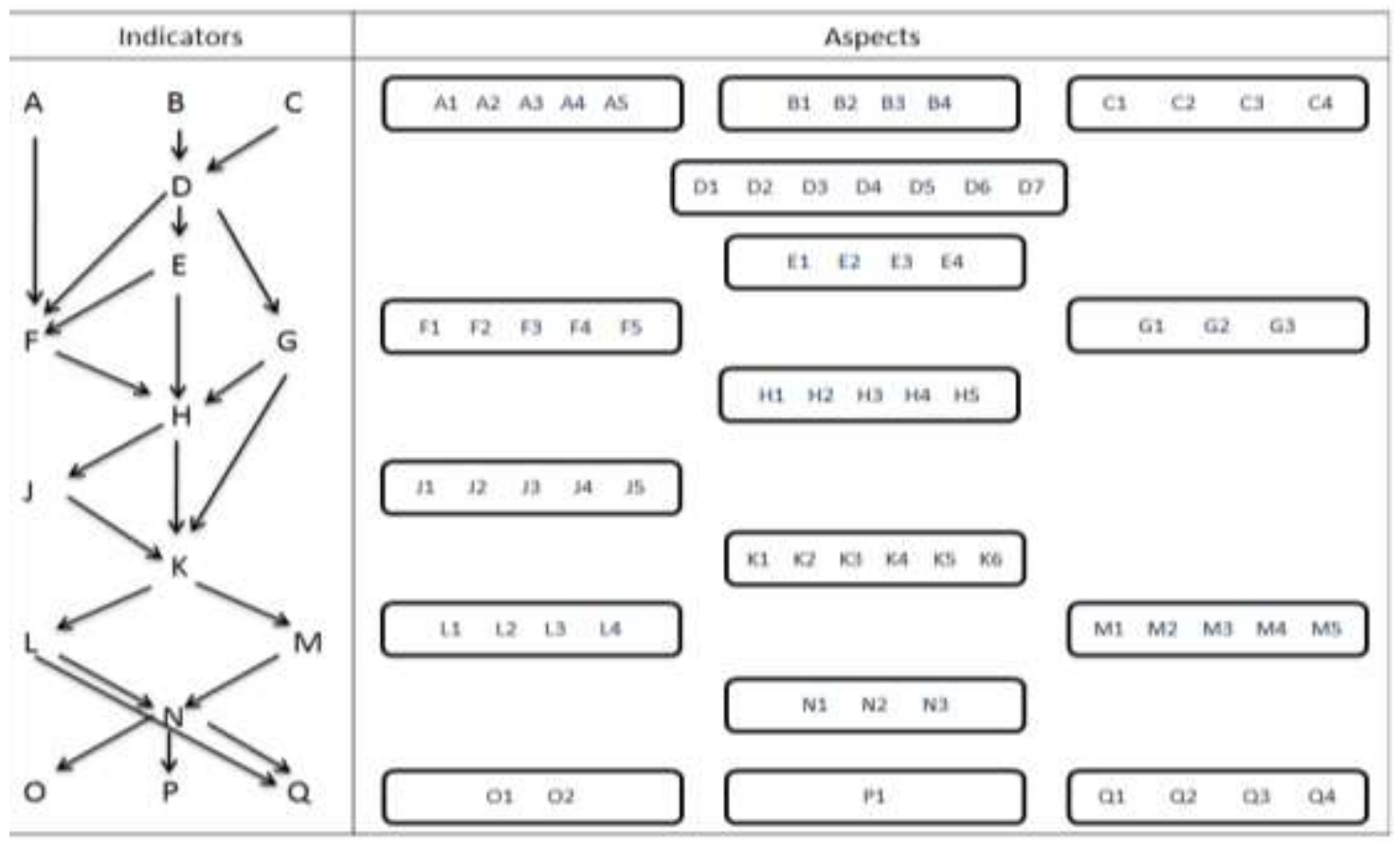

Figure 3.The structure of the creative process model.

The way of thinking relating to a creative activity is not linear in time. It happens in discontinuous flashes and jumps between aspects of the indicator process (see Figure 2, Figure 3 and Figure 4) as we identified it. At the "same time" ideas about other aspects of daily life or other problems are also happening and some of these may relate to the creative action, but most of them not. So the diagram we propose is created over some time period with several trial and error restarts of parts of the process. The level of "concentration" on the creative process is important for its smooth and rapid development. The really creative moments are probably unpredictable spread out in the time-evolution of the process. This creates extra influence of the uncertainty principle or fuzziness and is responsible for the creative idea to grow in interspersed periods sometimes resulting in a loss of clarity but also allowing progressing with giant leaps at certain moments, then recognized as moments of "inspiration". Real time repetitions of parts of the process in the construction of model, e.g. trial and error corrections have been omitted from our description. 


\begin{tabular}{|c|c|c|c|}
\hline I & Aspects & I & Aspects \\
\hline A & $\begin{array}{l}\text { A1-Level of self-awareness; A2-Historical } \\
\text { development of ego; A3- "Stability" of ego; A4- } \\
\text { Dominance of strength of ego }\end{array}$ & B & $\begin{array}{l}\text { B1-Level of awareness; B2-Broadness of process of } \\
\text { awareness; B3-Fluctuations in awareness; BA-Growth } \\
\text { capacity }\end{array}$ \\
\hline c & $\begin{array}{l}\text { C1-Structural properties (historic build-up); C2- } \\
\text { Memory accessibility; C3-Growing capacity } \\
\text { (learning capacity); C4--Quality (correctness) of } \\
\text { pre-knowledge }\end{array}$ & D & $\begin{array}{l}\text { D1-Methods of observation; D2-Definition of parameters } \\
\text { of observation; D3-Definition of clusters of observation; D4- } \\
\text {-Clarity and strength of observation; D5-Definition of } \\
\text { restrictions and limitations on observations; D6-Use of } \\
\text { structural pre-knowledge; D7-Ability to extend pre- } \\
\text { knowledge in the process of observation }\end{array}$ \\
\hline E & $\begin{array}{l}\text { E1-Analysis of time-evolution of learning } \\
\text { process; E2-Implementation evolution in pre- } \\
\text { knowledge (via the observations); E3-Analysis of } \\
\text { structural properties in the evolution of the } \\
\text { learning process; E4-Abstract analysis of } \\
\text { parameters and variation in time of them }\end{array}$ & $F$ & $\begin{array}{l}\text { F1--Role of self-awareness in learning process; F2- } \\
\text { Personality effects on learning process; F3--Analysis of } \\
\text { parameters in observation; F4-Personal choice in clustering } \\
\text { of observations; F5-Personal interpretation of restrictions } \\
\text { on observations }\end{array}$ \\
\hline G & $\begin{array}{l}\text { G1-Developing understanding about the } \\
\text { "reality" of the observations; G2-Recognition of } \\
\text { abstract structure (as discovered in B); G3-- } \\
\text { Placing the object in context with interpretations } \\
\text { of parameters, and restrictions and limitations }\end{array}$ & H & $\begin{array}{l}\text { H1-Observations relates to the new insight about reality of } \\
\text { object; H2-Abstract analysis of evolution of learning } \\
\text { process; H3-Abstract manipulation of the structural } \\
\text { properties; H4-Constructing experiments for actions on } \\
\text { objects; H5-improving or deleting conditions on parameters } \\
\text { or restrictions on the "real" object }\end{array}$ \\
\hline J & $\begin{array}{l}\text { J1-Redefining object, changing parameters. } \\
\text { restrictions; } 12 \text {-Inventing changes in structural } \\
\text { properties; } 13 \text {--Personalization of fantasy objects } \\
\text { derived (using A); } 14 \text {-Trving to relate deformed } \\
\text { object with the object from pre-knowledge; } 15 \text { - } \\
\text { Transferring structures or other properties from } \\
\text { related object (interaction pre-knowledge- } \\
\text { fantasy objects) }\end{array}$ & K & $\begin{array}{l}\text { K1-"Abstract realization" of fantasy object; K2- } \\
\text { Interpretation of the learning process (and its evolution) on } \\
\text { the realizability of fantasy object; K3-Redefining parameters } \\
\text { and restrictions at the fantasy objects; K4-Restructuring. } \\
\text { variation of the structural properties on the fantasy object; } \\
\text { K5--Constructing thought experiments on the fantasy } \\
\text { object; } K 6 \text {-Predicting properties of a possible realization of } \\
\text { the fantasvobject (eventually iterated after application of G. } \\
\text { and } \mathrm{H}_{\text {.) }}\end{array}$ \\
\hline L & $\begin{array}{l}\text { L1-Expressing the fantasy object, first try-out; } \\
\text { L2--iteration of the expression till final form; } 13 \text {-- } \\
\text { Interaction with personality: L4-Available tools } \\
\text { and capabilities (pre-knowledge) or development } \\
\text { of new tools and abilities (on fantasy level) }\end{array}$ & $M$ & $\begin{array}{l}\text { M1--Realization of fantasy object in "natural"; M2-- } \\
\text { Interaction of new object with existing frame; M3-- } \\
\text { Observing new interreletions for the new embedded object; } \\
\text { M4--Evaluation of new correctness of new "reality"; M5-- } \\
\text { Available tools and capabilities (pre-knowledge) to } \\
\text { development of new tools and abilities (in realized level) }\end{array}$ \\
\hline $\mathbf{N}$ & $\begin{array}{l}\text { N1-Social impacts on personal expression; N2- } \\
\text { Socialinteraction via the expression; N3--Social } \\
\text { recognition }\end{array}$ & 0 & $\begin{array}{l}\text { O1-Effect of creation on personality: } 02 \text {-Augmented } \\
\text { motivation }\end{array}$ \\
\hline $\mathbf{P}$ & $\begin{array}{l}\text { P1-Effect of creation on observation of universe } \\
\text { and society }\end{array}$ & $\mathbf{Q}$ & $\begin{array}{l}\text { Q1-..Questions concerning applications of new creation; } \\
\text { Q2--Problems about new interrelations; Q3-Deriving new } \\
\text { fantasyobject; Q4-Extra motivation from creation (social } \\
\text { recognition) }\end{array}$ \\
\hline
\end{tabular}

Figure 4. Aspects of the creative process model. "I"="Indicators".

Together with Figure 3 and Table 1 this set of arrows describes the essence of our theoretical description of the creative process. The reader should not see this as a set of rather random arrows, but should investigate carefully the meaning of each given relation. We stress the following important remarks. 1) The aspect relation is not transitive; arrows do not represent a causal relation, so composition of two arrows is not necessarily an arrow. We did not list all arrows but restricted to those most clear from our theoretical understanding of the process. The aspect relation induces a causal relation on the ingredients, hence it is in some sense pre-causal, i.e. if we have $A<B<C$ for ingredients then there have to be arrows from A (A) to A (C), not necessarily compositions of arrows via A (B). We focused on arrows between aspects on consecutive levels and did not claim completeness, but deep enough to deal with complex processes. 2) We could define weights for the aspects based on our actual understanding but we preferred not to do this now. First it would be better to have results from a lot of practical tests before doing the weighing but also the case where all aspects have the same weight allows the most complex processes from the psychological point of view. Indeed this is most clear by looking at an extreme weighing obtained by having in each set of aspects one which is far more important than others (a factor 100, 1000, or even more say). By the logic of the process important aspects have to appear in the relation, otherwise they would not be important, and important aspects relate to important aspects for the same reason. Therefore, since we have a unique important aspect for every ingredient, in this case the aspect relation would be, with very high probability, almost a copy of the ingredient relation, up to a few arrows of neglectable importance. In such weighing we would therefore not see the influence of non-causality in the process. Thus, even though the unweighted case is making the mathematical formalism of the inducing of the ingredient relation very easy (a causal relation from A to $\mathrm{B}$ is given exactly when less arrows go from aspects of B to aspects of A than conversely) it is the situation where the 
complexity of the process described may be maximal.

Table 1. Arrows on the Aspect Level in the Creative Process

\begin{tabular}{|c|c|}
\hline Category & Arrows \\
\hline Positive & (B1, D1), (C1, D1), (B2, D2), (C4, D2), (B4, D3), (C1, D5), (C1, D6), (C2, D7), (C4, D7), (D2, E1), (D4, \\
\hline \multirow[t]{13}{*}{ Arrows } & E1), (D4, E2), (B1, E3), (C1, E3), (D2, E4), (B1, F1), (B2, F1), (A1, F1), (A2, F2), (A4, F2), (C1, F3), \\
\hline & (C3, F3), (A3, F4), (A2, F5), (D2, G1), (D3, G1), (E4, G1), (D2, G2), (D3, G2), (D4, G2), (E3, G2), (D2, \\
\hline & G3), (D3, G3), (D4, G3), (E4, G3), (C1, G3), (C2, G3), (F1, H1), (F2, H1), (F3, H1), (F4, H1), (F5, H1), \\
\hline & (F3, H4), (F5, H5), (E1, H1), (E2, H1), (E3, H1), (E4, H1), (E3, H2), (E4, H2), (E4, H4), (G1, H1), (G2, \\
\hline & H1), (G3, H1), (G1, H2), (G2, H2), (G3, H2), (G1, H3), (G2, H3), (G3, H3), (G3, H4), (G3, H5), (H1, J1), \\
\hline & (H2, J1), (H3, J1), (H4, J1), (H5, J1), (H3, J2), (H4, J2), (H5, J2), (H1, J3), (H3, J3), (H5, J3), (H2, J4), \\
\hline & (H3, J4), (H5, J4), (H3, J5), (H5, J5), (J1, K1), (J2, K1), (J3, K1), (J4, K1), (J5, K1), (J1, K2), (J3, K2), \\
\hline & $(\mathrm{J} 1, \mathrm{~K} 3),(\mathrm{J} 2, \mathrm{~K} 4),(\mathrm{J} 4, \mathrm{~K} 5),(\mathrm{J} 4, \mathrm{~K} 6),(\mathrm{J} 5, \mathrm{~K} 6),(\mathrm{H} 1, \mathrm{~K} 1),(\mathrm{H} 2, \mathrm{~K} 1),(\mathrm{H} 3, \mathrm{~K} 1),(\mathrm{H} 4, \mathrm{~K} 1),(\mathrm{H} 5, \mathrm{~K} 1),(\mathrm{H} 1$, \\
\hline & K2), (H2, K2), (H5, K2), (H5, K3), (H3, K4), (H4, K5), (H3, K6), (G1, K1), (G2, K1), (G3, K1), (G3, \\
\hline & K2), (G3, K3), (G2, K4), (G3, K5), (G1, K6), (K1, L1), (K2, L1), (K3, L1), (K4, L1), (K5, L1), (K6, L1), \\
\hline & (K3, L2), (K4, L2), (K1, L3), (K6, L4), (K1, M1), (K2, M1), (K3, M2), (K4, M3), (K5, M3), (K6, M4), \\
\hline & (K6, M5), (K6, N1), (L3, N1), (K6, N2), (K6, N3), (L2, N3), (N1, O1), (N2, O2), (N1, P1), (N2, P1), (N3, \\
\hline & P1), (M3, Q1), (M3, Q2), (L2, Q3), (M4, Q3) \\
\hline Negative & (D4, C1), (D5, C3), (E1, D3), (G1, D7), (G2, D1), (F4, C1), (H4, G1), (J4, H1), (J4, H5), (K6, H1), (K6, \\
\hline Arrows & G3), (K5, H4), (K3, H4), (M5,G3), (M4, C4), (M2,D7), (Q2, C4), (P1, B2), (O2, A1), (O1, A3), (O1, A4) \\
\hline
\end{tabular}

Note. "Positive Arrows" = $\mathbf{q}_{+}$; "Negative Arrows"= q, not $\mathbf{q}_{+}$.

\subsection{Time Evaluation in the Creative Process}

We look at the process from its finalized form, that is we see a set of ingredients with a partial order (or a more general relation if wanted) covered by finite sets of aspects describing the ingredients completely (or as good as possible) with aspect relations defining in some sense (to be clarified later) the underlying relation between ingredients which are shadow objects, i.e., satisfactory approximations of some reality. We also see how this blue-print evolved in time with temporary shadow objects, temporary sets of aspects and relations; this time evolution can be encoded by giving to each component in the final picture a function of the time interval for the process to the interval of numbers between 0 and 1 , expressing for example the percentage of realization of the component at that moment in time. The learning process based on the shadow objects as ingredients is thus evolving in time growing in a discrete way within the final form framework step by step realizing each final component at certain (different) moments in time.

In order to give information on the way the process evolves in time we may start from the given final diagram and introduce intensity functions, i.e. for every moment $t$, a function it $: t \rightarrow[0.1]$ giving for every aspect a in $t$ the value it (a), the intensity of a at moment $t$, being the percentage expressing how much of the aspect a is present at moment t. Each value it (a) is growing with $t$ until it reaches $100 \%$ (i.e. 1 in $[0,1]$ ). Theoretically we consider the process finished when all reach intensity 1 . Given a certain (linearly ordered) path filling up the diagram in a certain time there are intensity functions corresponding to this and these functions depend heavily on the chosen path. The intensity functions reflect therefore the way a certain individual is going through the development of the process. In pure learning processes there may be identified a theoretical intensity corresponding to the development chosen by the developer of 
the study material (course, oral communication, etc.). In a creative process there may not be a theoretical intensity as the whole process depends much more on the personal input of the individual going through the process.

To find out about the existence of the "arrows", aspects and ingredients in the process, the planned questionnaire can be an adequate tool; to obtain information on the time evolution of the process diagram, i.e. to get an idea about the intensity functions, is much harder. Obviously this implies that the path chosen by individual to order linearly (in time) the logical connections in the process has to be known, from knowledge of the path one may estimate the intensity functions but individual differences are always possible. This requires much deeper investigations into the psyche and personality of the individual; it is still possible to find a measuring method for this but it will be harder and probably less precise.

\subsection{Measurability}

We supposed that there are lots of arrows among indicators or aspects. The planned questionnaires are used to test every arrow. Then we could give a value to every aspect according to the distinct importance. Based on the evaluation of the questionnaires and aspects weight, the rationality and the appropriateness of the model could be calculated (see definition part). In practice we plan to first study some important arrows based on an educated guess, the outcome of the tests we construct should provide a final proof of the correctness of the basis of our educated guess on the importance of the arrows we focused on.

\subsection{Definitions of Indicator and Aspects}

The three basic indicators of the creative process in our model are ego, awareness and pre-knowledge (Figure 2). The whole learning process is based on a cluster of observations. We call the process from every observation to a temporary shadow object a minimal learning process. The minimal learning processes could be seen as the micro-structure of the learning process. In a creative process there is a part of the diagram rooted in pre-knowledge, we call this the factual sub-process, and one rooted in the ego, called the emotional sub-process. Conscious and unconscious brain activities are present in both sub-processes. On the right of the diagram(pre-knowledge), the factual side, there is more conscious analysis and use of some (factual) intuition, while on the left (ego), the emotional side there is more influence from unconscious activities, e.g. leading to "creative intuition".

\subsubsection{Ego}

To our model of the creative process, ego represents many aspects of personal characteristics, such as emotion, personality, motivation, and ego-strength. In detail, it includes four aspects: level of self-awareness, historical development of ego, "stability" of ego, and dominance or strength of ego. Self-awareness is that individuals are aware of the existence of oneself by perception. At first, individuals concentrate on what they think about the outer world, and then based on the information about the outer world, individuals have reflection or retrospection of oneself, something like meta-cognition. "Historical development of ego" tends to focus on life stories of individuals. The aspect of "stability" of ego fits the dimension of Neuroticism in Eysenck Personality Questionnaire (EPQ) (Eysenck \& Eysenck, 1975) well. The dominance of strength of the ego, which might be a crucial aspect of creativity, means that the individual has the ability to control oneself, focus on a certain thing for a long time, and has the capacity to adjust the self-expectation (e.g., the need of oneself and the restrictions of circumstance). Runco (1996) stated that ego strength "will allow the individual to stand up to pressures to conform, and this may be vital because interpretive transformations are by definition personal, at least initially, and therefore can be rather unconventional"(p. 5). From this point of view, ego strength may be a crucial aspect of creativity.

\subsubsection{Awareness}

Awareness is defined as a human's or animal's perception and cognitive reaction to a condition or event in biological psychology. In our line of research, four aspects are included in this indicator: level of awareness (compare with fluctuations in awareness), broadness of process of awareness, fluctuations in awareness, and growth capacity.

\subsubsection{Pre-knowledge}

Pre-knowledge is an indicator that is closely related to intelligence. It includes all aspects of knowledge accumulated by individual. In addition to the latent and dominant pre-knowledge, it also includes structure, breadth, depth and correctness. If we explain this part using the SOI model, content and product, these two dimensions could well-explain the pre-knowledge. To the learners, two other aspects are also the key points-memory accessibility and learning capacity. These two aspects correspond to the dimension of operations of the SOI model, which shows that memory and cognition are greatly involved.

\subsubsection{Observation of Object}

An observer begins the study of the object by observing it. The nature of the object determines which senses are 
effectively used during the observation. There are two kinds of object, concrete object and abstract object. The interpretation of the observer is carried out via a complex biological system, which could also be named "thinking". The original observation may be enriched by information obtained from new observations. According to this, different descriptions from different points of view may be obtained. Thus we obtain clusters of observations which enhance the speed of the study and allowing a higher level of "accuracy" or "correctness" of observation. The "accuracy" or "correctness" could be defined as the level of correspondence between the image of object in the observer's mind and the real object. Based on the theories above, this indicator includes seven aspects: methods of observation, definition of parameters of observation, definition of clusters of observation, clarity and strength of observation, definition of restrictions and limitations on observations, use of structural pre-knowledge, and ability to extend pre-knowledge in the process of observation.

\subsubsection{Abstract Analysis}

As stated before, our concept of learning process is one of self-structuring processes. In some sense the shadow object is an abstraction of the study object. The abstract shadow object is more flexible and we can manipulate it in many ways. The relation one discovers are logical connections between temporary shadow objects and they become ingredients of the learning process. This indicator includes four aspects: analysis of time-evolution of the learning process, implementation of evolution in pre-knowledge (via the observations), analysis of structural properties in the evolution of the learning process, and abstract analysis of parameters and variation in time of them.

\subsubsection{Meta Learning}

This indicator is closely related to the individual characteristics. Interest and curiosity arouse strong motivation to express oneself or figure out something. Fantasy and imagination greatly enrich the shadow objects and allow the observer to find connections between different images where this is not no evident. There are five ingredients included in this indicator: role of self-awareness in learning process; personality effects on learning process; analysis of parameters in observation; personal choice in clustering of observations; and personal interpretation of restrictions on observations.

\subsubsection{Insight of Given Object}

Insight is created by recombining understanding and the observations of reality. This indicator is divided into three important aspects: developing understanding about the "reality" of the observations, recognition of abstract structure, and placing the object in context with interpretations of parameters, restrictions and limitations.

\subsubsection{Abstract Manipulation of Definitions and Restrictions}

It is the decisive indictor for creativity. We propose five aspects of this indicator: observations related to the new insight about reality of object; abstract analysis of the evolution of learning process; abstract manipulation of the structural properties; constructing experiments for actions on objects; and improving or deleting conditions on parameters or restrictions on the "real" object. These items could help an individual to have an in-depth understanding about the real object and the situation of the reality. If we view this indicator in the context of Wallas (1926) four-stage model, this indicator is partially in incubation stage but mainly in preparation stage.

\subsubsection{Creation of Fantasy Object and Personalization of Object Involving Emotion}

Following the previous indicator, this is the other indicator that should be underscored in a creative process. We named the transformation process happening in these two indicators as "creative transformation" (creative transformation abilities will be illustrated in another paper). Feldman, Marrinan, and Hartfeldt (1972) defined transformational power as "the extent to which a given response represents the production of new forms rather than improves upon existing forms, the extent to which the apparent constraints of the stimulus situation are overcome, but overcome in a highly appropriate fashion, and the extent to which the product generates additional thoughts in the observer" (p. 336). Thus transformational power placed great emphasis on the new forms distinct from the existing forms. On the other hand, the transformation process reflects individual characteristics. We strongly agree with Runco's (1996) viewpoint that "transformational capacities underlie the construction of personal interpretations" (p. 6). Research of Tobias (1994) suggests the relationship between interest and learning. The author points out that "working on interesting, compared to neutral materials may engage deeper cognitive processing, arouse a wider, more emotional, and more personal associative network, and employ more imagery" (p.50). In such a process, it shows that individuals merge themselves into the learning process. They have their own strong feelings and insights about the object or knowledge which we called as "personal interpretation". It is an excellent basic process to create new ideas or solve problems in a creative way.

This indicator per se contains substantially changes which are showed in the following five steps: redefining object, changing parameters and restrictions; inventing changes in structural properties; personalization of fantasy objects 
derived; trying to relate deformed object with the object from pre-knowledge; and transferring structures or other properties from related object. Fantasy object plays a key role in this indicator and as mentioned in foregoing paragraph, it is involved in the primary-process thinking which can also be seen as affect-laden cognition. Due to this, we postulate that this indicator uses a high emotional involvement.

\subsubsection{Analysis of Possible Realization of Fantasy Objects}

This is a pivotal step to satisfy the trait of appropriateness/usefulness of creativity. A 6-step continuous procedure occurs in this indicator: "abstract realization" of fantasy object; interpretation of the learning process (and its evolution) on the realization of fantasy object; redefining parameters and restrictions at the fantasy objects; restructuring, variation of the structural properties on the fantasy object; constructing thought experiments on the fantasy object; and predicting properties of a possible realization of the fantasy object. Creative products are formed only after this process in a variety of expressions.

\subsubsection{Artistic Expression of Fantasy Object}

In terms of emotional expression, five aspects will contribute the final product: expressing the fantasy object, first try-out; iteration of the expression till final form; interaction with personality; available tools and capabilities (pre-knowledge) or development of new tools and abilities (on fantasy level).

\subsubsection{Natural and Scientific Realization of Fantasy Object}

As a form of rational discovery, five aspects are included in this indicator: realization of fantasy object in a "natural" context; interaction of new object with existing frame; observing new interrelations for the new embedded object; evaluation of new correctness of new "reality"; available tools and capabilities (pre-knowledge) to development of new tools and abilities (in realized level).

\subsubsection{Social Input and Social Recognition}

We may state that everyone is capable of creative activity and motivation, concentration, intelligence may enhance it. Moreover, the recognition of the results by others (the society) is also a very important factor for the normal development of creativity that can also have a steering effect. Lack of recognition of creative activities may lead to a diminished activity or even to abnormal forms of creative thinking (like withdrawing in a fantasy world). The recognition of creative activities may also lead to a form of contentment or happiness: your creation "benefits" society. Lack of recognition may lead to frustrations and bitterness. The final "new awareness" or changes in the personality may then depend on the reactions of the social environment towards creative activities. Three aspects should be taken into consideration in this indicator: social impacts on personal expression; social interaction via the expression; and social recognition.

\subsubsection{Augmented Ego}

At the end of the creativity cycle, augmented ego will be achieved in two aspects, effect of creation on personality and augmented motivation.

\subsubsection{New Awareness}

Individual is supposed to benefit from the effect of the creation on the observation of the universe and the society.

\subsubsection{New Research Problem}

This indicator suggests that a new learning process or creative process will occur after this. It was described by four aspects: questions concerning applications of the new creation; problems about new interrelations; deriving new fantasy object; and extra motivation from the creation (social recognition).

\section{Discussion and Conclusions}

\subsection{Our learning Process on the Creativity Process}

In the model we assume that the relation (denoted by arrows) on the level of process indicators is a partial order, in particular we assume it is given by causality. This is very logical if we describe the "aware" part of the process, which is the part we are conscious of. In this case the relation is transitive, so composition of two arrows is again an arrow, so we do not have to draw it as an extra one. However, we did draw an extra arrow occasionally if it seemed a particularly interesting one. On the level of aspects the relation is not transitive, hence not given by pure causality, so an arrow on this level does not necessarily mean an implication, also we have to draw all arrows, as sometimes composition of arrows does not correspond to an arrow. Here there may also be "unconscious" effects yielding arrows that cannot be explained by causality, we may notice the effect but cannot explain where it comes from. This is intrinsically connected to the effect of fantasy. It relates to "random" thinking and to the possible existence of free will even at the unconscious level excluding a "deterministic" interpretation by causality. Hence it is extremely important not to assume a causal 
ordering on the aspect level while it is harmless to assume this on the indicator level where a clear distinction between cause and consequence is present because this is the way we observe things! Thus the indicator level of the process may be thought of as the observed process while the aspect level provides a first description of the internal process in the mind including possibly effects from an unconscious process involving non-causal interactions. Therefore arrows on the aspect level going in the wrong direction when compared to the arrows on the underlying ingredients, even if they do not contribute really to the relation on ingredients, are particularly interesting. They may be even contra-intuitive and may be hard to be described; we believe a lot deeper research in the picture on the aspect level is necessary in order to fully understand the "wild" characteristics of the effects of fantasy perhaps stemming from the unconsciousness.

\subsection{Understanding and Insight}

We defined understanding as the level of knowledge about the micro-structure of the process and its clusters-process, while insight was the combination of this knowledge with interpretation of the reality. Based on the theory of the factual sub-process and the emotional sub-process, there is more understanding on the factual side, understanding of the emotional sub-process will be less conscious, we call it creative understanding.

In fact, the reality corresponding to the emotional sub-process should be the fantasy object created by the individual and these need not reflect the properties of the factual reality it is derived from. Indeed, the more creative the individual is the more discrepancy there may be between fantasy objects and factual reality. "Creative understanding" about the micro-structure of the emotional sub-process may be less conscious even if its combination with interpretation of the fantasy objects leads to "creative insight". The understanding and insight of the factual sub-process have to be combined with the creative understanding and creative insight in order for the creativity to be related to reality. The difference between understanding and creative understanding is fundamental. If an individual fails to recognize the difference then his world will be a strange mix of fantasy and reality and in some extreme cases he may not distinguish clearly between them, for example, the reality value of a film or the news on TV may be blurred. It may be that our senses are somewhat specialized in the emotional or factual direction; hearing and sight are more factual whereas smell and taste are probably more emotional, that means that observations via these senses fit more in the left or right part of the process diagram for creative processes. The patterns of brain activity for smell and taste seem to be random, i.e. unique for each individual, whereas these for sight seem to be rather standardized. The sweet taste of revenge or the bitter taste of defeat seems to refer to emotions linked to taste.

\subsection{Fantasy and Imagination}

Imagination allows to form an image or a shadow object without there being any reality behind it. There are different types or levels of fantasy. First the fantasy necessary to define induced abstract objects and properties of observed objects and to see their inter-relations on the abstract level; secondly the fantasy necessary to draw from seemingly unrelated facts in the pre-knowledge in order to analyze an object further, this seems to depend on the possible use of "long" links in the brain and may be related to" wandering" of thoughts; the fantasy inherent in the creation of relations between fantasy objects yielding a fantasy-logic having a coherence within the fantasy (this is like telling a fantasy or science-fiction story); the creative fantasy that leads to fantasy-insight, that is a relation between fantasy and the reality leading to the creation of inventions or art masterpieces. The first three types may be trained and are important in learning processes and education systems of any type.

\subsection{Implication for Didactics}

Firstly there is the effect of the "uncertainty principle", i.e., the time ordered digestion of the graph-likes the structure of the logical connection between ingredients. The teacher should be aware of the logical structure in the course include repetitions dealing with the bifurcation points in the graph of the partial order relation. Secondly, the aim is to reach understanding (the knowledge of micro-structure of the process) not just factual knowledge. In mathematics this means that giving proofs is essential for reaching understanding. Thirdly, we have to involve creativity in the learning process because that is essential for creating interest and motivation. So the structure of the teaching should aim at the stimulation of activities in the creative transformation. This can be done by using different teaching methods, e.g., research lectures, discovery lectures, and error detection lectures. Finally the level of the intensity function for each ingredient can be tested by regular evaluation moments (also by self-evaluation) where all (or at least the most important) ingredients are tested on the level of understanding. The score and its evaluation over the regular tests give an idea about how the learning process is growing in the student. When questions are put into the right way, that is really testing the "understanding" of the ingredients and aspects, the results of the test can be the basis of extra attention of the teacher to certain problems encountered by the students. The regular testing is itself a learning process for the teacher on the evaluation of the students learning processes. The tests should also test the creative activity (for example by asking a few open ended questions). 


\subsection{Limitations and Future Research}

All the foregoing points out that we are putting the first steps in the learning process on the creative process again fitting in an ingredient-aspect deformation process that we will not try to analyze in-depth here; but it is important to mark this study as the beginning leaving a lot of future research possible. In particular the model allows a very detailed study but we have to restrict to some essential points, therefore we shall not try to "measure" all aspects and ingredients with their interrelations but specialize to a few well-chosen relations where our questionnaire can shed enough light; in other words we should focus on some sub-process of the total process as far as validation checking will go but theoretically we present the full abstract picture.

\section{References}

Beghetto, R. A., \& Kaufman, J. C. (2007). The genesis of creative greatness: Mini-c and the expert performance approach. High Ability Studies, 18(1), 59-61. http://dx.doi.org/10.1080/13598130701350668

Colvin, S. S., \& Meyer, I. F. (1906). Imaginative elements in the written work of school children. Pedagogical Seminar, 13, 91. http://dx.doi.org/10.1080/08919402.1906.9943595

Davidson, J. E., \& Sternberg, R. J. (1984). The role of insight in intellectual giftedness. Gifted Child Quarterly, 28(2), 58-64. http://dx.doi.org/10.1177/001698628402800203

Eysenck, H. J., \& Eysenck, S. B. G. (1975). Manual of the Eysenck Personality Questionnaire (Junior and Adult). Kent, UK: Hodder \& Stoughton.

Feldman, D. H., Marrinan, B. M., \& Hartfeldt, S. D. (1972). Transformational power as a possible index of creativity. Psychological Reports, 30(1), 335-338. http://dx.doi.org/10.2466/pr0.1972.30.1.335

Finke, R. A., Ward, T. B., \& Smith, S. M. (1992). Creative cognition: Theory, research, and applications. Cambridge, MA: MIT Press.

Kaufman, J. C., \& Beghetto, R. A. (2009). Beyond big and little: The four C model of creativity. Review of General Psychology, 13(1), 1-12. http://dx.doi.org/10.1037/a0013688

Mednick, S. A. (1962). The associative basis of the creative process. Psychological Review, 69(3), 220-232. http://dx.doi.org/10.1037/h0048850

Mumford, M., \& Gustafson, S. (1988). Creativity syndrome: Integration, application, and innovation. Psychological Bulletin, 103(1), 27-43. http://dx.doi.org/10.1037//0033-2909.103.1.27

Rhodes, M. (1961). An analysis of creativity. Phi Delta Kappan, 42(7), 305-310.

Runco, M. A. (1996). Personal creativity: Definition and developmental issues. New Directions for Child and Adolescent Development, 1996(72), 3-30. http://dx.doi.org/10.1002/cd.23219967203

Ruscio, J., Whitney, D. M., \& Amabile, T. M. (1998). Looking inside the fishbowl of creativity: Verbal and behavioral predictors of creative performance. Creativity Research Journal, 11(3), 243-263. http://dx.doi.org/10.1207/s15326934crj1103_4

Sternberg, R. J. (2006). The nature of creativity. Creativity Research Journal, 18(1), 87-98. http://dx.doi.org/10.1207/s15326934crj1801_10

Sternberg, R. J., \& Davidson, J. E. (1982). The mind of the puzzler. Psychology Today, 16(6), 37-44.

Tobias, S. (1994). Interest, prior Knowledge, and learning. Review of Educational Research, 64(1), 37-54. http://dx.doi.org/10.3102/00346543064001037

Wälder, R. (1933). The psychoanalytic theory of play. Psychoanalytic Quarterly, 2, 208-224.

Wallas, G. (1926). The Art of Thought. New York: Harcourt, Brace \& Jovanovich.

\section{$(\mathrm{Cc}) \mathrm{BY}$}

This work is licensed under a Creative Commons Attribution 3.0 License. 